\title{
Charm Fragmentation and Excited Charm Meson Production
}

\author{
Olaf Behnke* \\ (on behalf of the ZEUS and H1 Collaborations) \\ DESY, Notkestrasse 85, Hamburg 22607, Germany \\ E-mail: obehnke@mail.desy.de
}

In the first part of the talk measurements were presented by $\mathrm{H} 1$ and ZEUS of the charm fragmentation into $D^{*}$ mesons at HERA, investigating the charm fragmentation function. Since these results were already presented at the EPS-HEP 2009 conference, the reader is referred to the corresponding proceedings [1]. In the remainder of this article we will focus completely on the second part of the talk, presenting a new ZEUS measurement [2] of the production of the excited charm mesons $D_{1}(2420)^{0}$ and $D_{2}^{*}(2460)^{0}$ in ep collisions. The masses, widths and helicity parameters of these resonances were determined and compared with previous measurements and theoretical expectations. The results can also be compared with new high precision measurements [3] by BABAR, which were presented at this conference. A good agreement between the ZEUS and BABAR results is observed.

35th International Conference of High Energy Physics - ICHEP2010,

July 22-28, 2010

Paris France

${ }^{*}$ Speaker. 


\section{Introduction}

The large charm production cross section and the well established ground state charm mesons $D$ and $D^{*}$ allow a search for excited charm meson states in $e p$ collisions at HERA. In a previous analysis of the production of such states [ $\left[\right.$ ], the orbitally excited states $D_{1}(2420)^{0}$ with $J^{P}=$ $1^{+}$and $D_{2}^{*}(2460)^{0}$ with $J^{P}=2^{+}$were studied in the decay modes: $D_{1}(2420)^{0} \rightarrow D^{* \pm} \pi^{\mp}$ and $D_{2}^{*}(2460)^{0} \rightarrow D^{* \pm} \pi^{\mp}, D^{ \pm} \pi^{\mp}$. The width of the $D_{1}^{0}\left(53.2 \pm 7.2(\text { stat. })_{-4.9}^{+3.3}(\right.$ syst. $) \mathrm{MeV}$ ) was above the world average value $20.4 \pm 1.7 \mathrm{MeV}$ [阿]. In addition, a study of the helicity angular distribution of the $D_{1}(2420)^{0}$ was consistent with some S-wave admixture in the decay $D_{1}^{0} \rightarrow D^{* \pm} \pi^{\mp}$, contrary to theoretical predictions [6] and to previous experimental results [?] which yielded a pure D-wave decay in this channel.

In this paper we repeat the analysis with an independent data sample of higher integrated luminosity. The new analysis is performed with the upgraded ZEUS detector, which included a Micro-Vertex Detector (MVD). The analysis was performed using data taken from 2003 to 2007, when HERA collided electrons or positrons at $27.5 \mathrm{GeV}$ with protons at $920 \mathrm{GeV}$. The data correspond to an integrated luminosity of $373 \mathrm{pb}^{-1}$. To maximise the statistics, events with both photoproduction (PHP) and deep-inelastic scattering were used in this analysis. Events produced in the PHP regime contributed $70-80 \%$ of the selected charm meson samples.

\section{Results}

$D^{* \pm}$ mesons were identified via the decay mode $D^{*+} \rightarrow D^{0} \pi_{s}^{+} \rightarrow\left(K^{-} \pi^{+}\right) \pi_{s}^{+}$, where $\pi_{s}$ is a low-momentum ("soft") pion due to the small mass difference, $\Delta M$, between $D^{*+}$ and $D^{0}$. The corresponding antiparticle decays were also measured. ${ }^{1}$ A clean $D^{*+}$ signal of $66,804 \pm 448$ events is seen (not shown here) in the $\Delta M$ distribution and all $D^{*+}$ candidates with $0.144<\Delta M<0.147 \mathrm{GeV}$ were used for the subsequent excited charm mesons analysis.

$D^{ \pm}$mesons were reconstructed from the decay $D^{+} \rightarrow K^{-} \pi^{+} \pi^{+}$with looser kinematic cuts than in the previous analysis [ [ significance, defined as $S=l / \sigma_{l}$, was used to reject combinatorial background, where the decay length $l$ is the distance in the transverse plane between the production point and decay vertex of the $D^{ \pm}$meson and $\sigma_{l}$ is the uncertainty of this distance. A clear $D^{ \pm}$signal of 53,902 \pm 446 events is seen (not shown here) in the $M\left(K^{-} \pi^{+} \pi^{-}\right)$distribution for events with significance $S>3$ and all such $D^{ \pm}$candidates with $1.85<M(K \pi \pi)<1.89 \mathrm{GeV}$ were used for the subsequent excited charm mesons analysis.

The $D_{1}(2420)^{0}$ and $D_{2}^{*}(2460)^{0}$ mesons were reconstructed in the decay mode $D^{*+} \pi^{-}$. For each excited charm meson candidate, the "extended" mass difference, $\Delta M^{e x t}=M\left(K \pi \pi_{s} \pi_{a}\right)-$ $M\left(K \pi \pi_{s}\right)$, was calculated where $\pi_{a}$ is the extra pion combined with the $D^{*}$. Fig.1 (upper plot) shows the invariant mass $M\left(D^{*+} \pi_{a}\right)=\Delta M^{e x t}+M\left(D_{P D G}^{*+}\right)$, where $M\left(D_{P D G}^{*+}\right)$ is the nominal $D^{*+}$ mass [5]. A clear signal of the combined $D_{1}^{0} / D_{2}^{* 0}$ mesons is seen. The histogram is the distribution for wrong-charge combinations $M\left(D^{* \pm} \pi^{ \pm}\right)$.

The $D_{2}^{*}(2460)^{0} \rightarrow D^{+} \pi^{-}$decay mode was reconstructed by calculating the "extended" mass difference $\Delta M^{e x t}=M\left(K \pi \pi \pi_{a}\right)-M(K \pi \pi)$. Fig.1 (lower plot) shows the invariant mass $M\left(D^{+} \pi_{a}\right)=$

\footnotetext{
${ }^{1}$ Hereafter, charge conjugation is implied.
} 

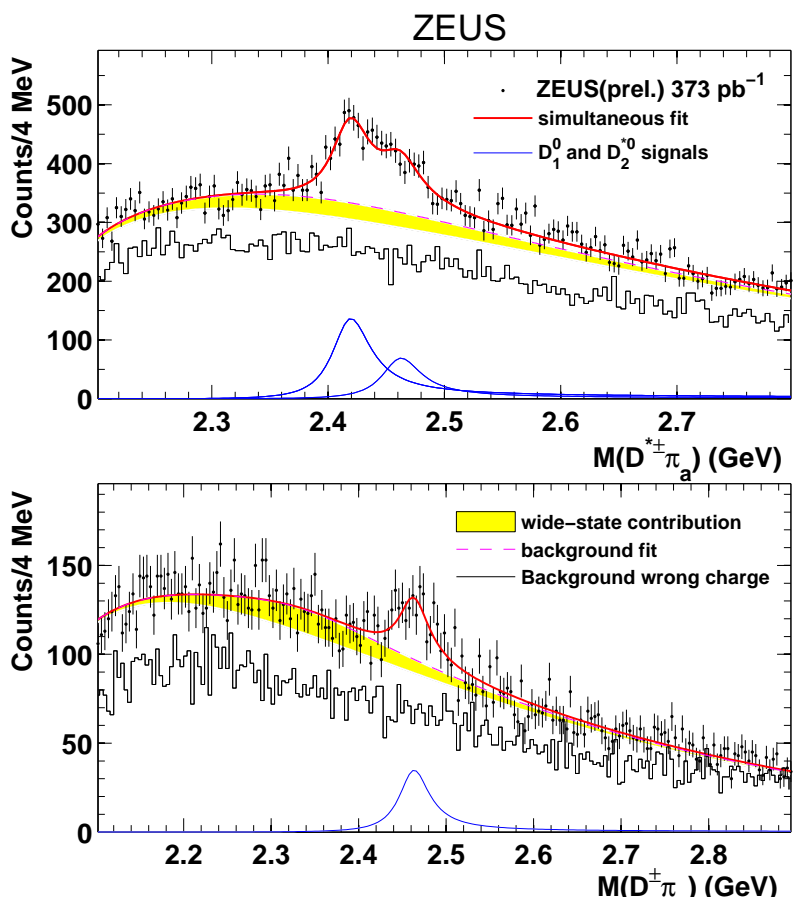

Figure 1: The mass distributions (dots) $M\left(D^{* \pm} \pi_{a}\right)$ (upper plot) and $M\left(D^{ \pm} \pi_{a}\right)$ (lower plot). The histograms are the corresponding distributions for wrong-charge combinations. The solid curves are the result of a simultaneous fit to $D_{1}^{0}$ and $D_{2}^{* 0}$ (upper plot) and to $D_{2}^{* 0}$ (lower plot) plus background (dashed curves). Contributions from the wide states $D_{1}(2430)^{0}$ and $D_{0}^{*}(2400)^{0}$ are shown as shaded bands.

$\Delta M^{\text {ext }}+M\left(D_{P D G}^{+}\right)$, where $M\left(D_{P D G}^{+}\right)$is the nominal $D^{+}$mass [5], for events with significance $S>3$. A clear $D_{2}^{* 0}$ signal is seen. The histogram is the distribution for wrong-charge combinations $M\left(D^{ \pm} \pi^{ \pm}\right)$.

To distinguish between $D_{1}^{0} / D_{2}^{* 0} \rightarrow D^{* \pm} \pi^{\mp}$, the $D_{1}^{0}$ and $D_{2}^{* 0}$ helicity angular distributions were used. These can be parametrised as $d N / d \cos \alpha \approx 1+h \cos ^{2} \alpha$, where $\alpha$ is the angle between the $\pi_{a}$ and $\pi_{s}$ momenta in the $D^{*+}$ rest frame and $\mathrm{h}$ is the helicity parameter, predicted [5] to be $\mathrm{h}=3$ for $D_{1}^{0}$ and $\mathrm{h}=-1$ for $D_{2}^{* 0}$. To extract the $D_{1}^{0}$ and $D_{2}^{* 0}$ masses, widths and yields, a simultaneous fit was performed on the $M\left(D^{+} \pi_{a}\right)$ distribution (Fig.1 lower plot) and the $M\left(D^{* \pm} \pi_{a}\right)$ distributions in four helicity bins (not shown here). Each resonance was fitted to a relativistic D-wave Breit-Wigner function [ 4 . Yields of the three signals, the $D_{1}^{0}$ and $D_{2}^{* 0}$ masses and the $D_{1}^{0}$ width and helicity, $h$, were free parameters of the fit. The $D_{2}^{* 0}$ width was fixed to the PDG value of $43 \mathrm{MeV}$ [ [] and $h\left(D_{2}^{* 0}\right)$ was fixed to the theoretical prediction [6] $h\left(D_{2}^{* 0}\right)=-1$. A separate fit to the $M\left(D^{+} \pi_{a}\right)$ distribution of Fig.1 (lower plot) with a single resonance and free width yielded a $\Gamma\left(D_{2}^{* 0}\right)$ consistent with the PDG value. Contributions from the wide states [5] $D_{1}(2430)^{0}$ and $D_{0}^{*}(2400)^{0}$ were added, respectively, to the $M\left(D^{* \pm} \pi_{a}\right)$ and $M\left(D^{+} \pi_{a}\right)$ fit with shapes described as relativistic S-wave BreitWigner functions. Their masses and widths were set to the PDG values [5] and their yields were taken, respectively, as 1.0 and 1.7 times the narrow states $D_{1}^{0}$ and $D_{2}^{* 0}$ 㺻. Systematic uncertainties of the measured values were estimated from variations of the selection cuts, fit ranges, background parametrisation, bin width of the fitted distributions and yields of the wide states. 
The results of the simultaneous fit (masses, widths and helicity parameters) are given in Tab. 1 and Fig. 1 (solid curves). The $D_{1}^{0}$ width, $\Gamma\left(D_{1}^{0}\right)=43.4 \pm 6.2$ (stat. $)_{-10.4}^{+7.3}($ syst. $) \mathrm{MeV}$, is above the PDG value [5] of $20.4 \pm 1.7 \mathrm{MeV}$, consistent with the previous ZEUS result [ [ parameter, $h\left(D_{1}^{0}\right)=3.5_{-1.0}^{+1.6}(\text { stat } .)_{-0.8}^{+2.0}($ syst .), is consistent with the pure D-wave prediction of $\mathrm{h}=3$.

The presented measurements can also be compared to new high precision results [3] by BABAR, which were presented at this conference. Among other parameters, BABAR measured the $D_{1}^{0}$ width to be $\Gamma\left(D_{1}^{0}\right)=31.4 \pm 0.5$ (stat.) \pm 1.3 (syst.) $\mathrm{MeV}$, which is also higher than the above-quoted PDG value. This result agrees well with the old [4] and new preliminary ZEUS results.

\section{Conclusions}

The full HERA data taken from 2003 to 2007 with an integrated luminosity $373 \mathrm{pb}^{-1}$ was used to study the production of the excited charm mesons $D_{1}(2420)^{0}$ and $D_{2}^{*}(2460)^{0}$. Nice signals are seen in both $D^{* \pm} \pi^{\mp}$ and $D^{ \pm} \pi^{\mp}$ decay modes. The measured $D_{1}^{0}$ and $D_{2}^{* 0}$ masses are in good agreement with the PDG values. The measured $D_{1}^{0}$ width is above the PDG value, consistent with a previous ZEUS measurement of an independent event sample. The measured $D_{1}^{0}$ helicity parameter allows for some $\mathrm{S}$-wave mixing in its decay to $D^{* \pm} \pi^{\mp}$. The result is also consistent with a pure D-wave hypothesis. The ZEUS measurements agree well with the recent high precision BABAR results [3].

\section{References}

[1] S. Fang [ H1 and ZEUS Collaboration ], POS (EPS-HEP 2009) 316

[2] ZEUS Collaboration, www-zeus.desy.de/physics/hfla/public/PublicPlots/Zeus-prel-10-016/dss_5.pdf.

[3] P. del Amo Sanchez et al. [The BABAR Collaboration], arXiv:1009.2076 [hep-ex].

[4] ZEUS Collaboration, S. Chekanov et al., Eur. Phys. J. C 60 (2009) 25.

[5] Particle Data Group, C. Amsler et al., Phys. Lett. B 667 (2008) 1.

[6] N. Isgur, M.B. Wise, Phys. Lett. B 232 (1989) 113; M. Neubert, Phys. ReV. A 245 (1994) 259.

\begin{tabular}{|c|c|c|c|}
\hline & HERA I & HERA II & PDG \\
\hline$M\left(D_{1}^{0}\right) \mathrm{MeV}$ & $2420.5 \pm 2.1 \pm 0.9$ & $2422.2 \pm 1.7_{-2.8}^{+1.2}$ & $2422.3 \pm 1.3$ \\
\hline$\Gamma\left(D_{1}^{0}\right) \mathrm{MeV}$ & $53.2 \pm 7.2_{-4.9}^{+3.3}$ & $43.4 \pm 6.2_{-10.4}^{+7.3}$ & $20.4 \pm 1.7$ \\
\hline$h\left(D_{1}^{0}\right)$ & $5.9_{-1.7-1.0}^{+3.0+2.4}$ & $3.5_{-1.0-0.8}^{+1.6+0}$ & \\
\hline$M\left(D_{2}^{* 0}\right) \mathrm{MeV}$ & $2469.1 \pm 3.7_{-1.3}^{+1.2}$ & $2465.0 \pm 3.3_{-2.9}^{+1.2}$ & $2461.1 \pm 1.6$ \\
\hline$\Gamma\left(D_{2}^{* 0}\right) \mathrm{MeV}$ & 43 fixed & 43 fixed & $43 \pm 4$ \\
\hline$h\left(D_{2}^{* 0}\right)$ & -1 fixed & -1 fixed & \\
\hline
\end{tabular}

Table 1: Results of the simultaneous fit for the masses $(M)$, widths $(\Gamma)$ and helicity parameters (h) of the $D_{1}^{0}$ and $D_{2}^{* 0}$ mesons. The first errors are statistical and the second errors are systematics. 